\title{
Software Selection in Manufacturing Industries Using a Fuzzy Multiple Criteria Decision Making Method, PROMETHEE
}

\author{
R. V. RAO, T. S. RAJESH \\ S. V. National Institute of Technology, Ichchanath, Surat, India \\ Email: ravipudirao@gmail.com
}

\begin{abstract}
This paper presents an effective decision making framework for software selection in manufacturing industries using a multiple criteria decision making method, Preference Ranking Organization Method for Enrichment Evaluations (PROMETHEE). The method is improved in the present work by integrating with analytic hierarchy process (AHP) and the fuzzy logic. Fuzzy logic is introduced to handle the imprecision of the human decision making process. The proposed decision making framework is practical for ranking competing software products in terms of their overall performance with respect to multiple criteria. An example is included to illustrate the approach.
\end{abstract}

Keywords: software selection, manufacturing industries, multiple criteria decision making, PROMETHEE, fuzzy logic

\section{Introduction}

Application of software in design and manufacturing processes is one of the resolutions many industries have resorted to in the $21^{\text {st }}$ century. This has been a result of increased complexity of products, globalization, rapid changes in technology, and so on. The idea was that the application of software would increase the competitive advantage of an industry. Various types of software are used by the manufacturing industries, such as product development process (PDP) software, product data management (PDM) software, product life-cycle management (PLM) software, enterprise resource planning (ERP) software, computer-aided design (CAD) software, computer-aided manufacturing (CAM) software, etc. The software used in various industries can be either COTS or in-house developed. COTS is acronym for commercial off-the-shelf, an adjective that describes software or hardware products that are ready-made, and available for sale to the general public. Given the high interest in motivation to the use of commercially available software in manufacturing industries, the evaluation and selection of COTS products is an important activity in software development projects. Selecting an appropriate COTS product is often a non-trivial task in which multiple criteria need to be carefully considered. With so many criteria to consider when selecting new software, help is needed to focus on the essentials and to avoid the software selection traps that many organisations fall into.
Clearly, software selection is not a well-defined or structured decision problem. The presence of multiple criteria (both managerial and technical) will expand decisions from one to many several dimensions, thus, increasing the complexity of the solution process. It seems obvious that the selection problem may not be solved simply by grinding through a mathematical model or computer algorithm. New approaches, which could handle multicriteria decision-making problems of choice and prioritization, to support these types of complex and unstructured selection problems are needed. Many decision makers select COTS products according to their experience and intuition. However, this approach is obviously subjective, and its weakness was addressed by Mikhailov and Singh [1].

During the past two decades, there has been a steady growth in the number of multiple criteria decision making (MCDM) methods for assisting decision making with multiple objectives. These MCDM methods allow decision makers to evaluate various competing alternative courses of action to achieve a certain goal. Santhanam and Kyparisis [2,3] proposed a non-linear programming model to optimize resource allocation and their model considered interdependencies between projects in the information system selection process. Carney and Wallnau [4] observed that there are almost as many perspectives on the topic of software evaluation as there are evaluation techniques. The authors developed some basic principles applicable for evaluation of commercial-off- 
the-shelf software. Sarkis and Sundarraj [5] discussed about various factors for strategic evaluation of enterprise information technologies. Badri et al. [6] presented a goal programming model to select an information system project considering multiple criteria including benefits, hardware, software and other costs, risk factors, preferences of decision makers and users, completion time, and training time constraints.

Morisio et al. [7] investigated COTS-based software development within a particular NASA environment, with an emphasis on the processes used. Fifteen projects using a COTS-based approach were studied and their actual process was documented. This process was evaluated to identify essential differences in comparison to traditional software development. The authors concluded that the main differences, and the activities for which projects require more guidance, were requirements definition and COTS selection, high level design integration and testing.

The analytic hierarchy process (AHP) was widely used by both researchers and practitioners in COTS selection processes [8-13]. Lai et al. [8] reported the results of a case study where the AHP technique was employed to support the selection of a multimedia authorizing system (MAS) in a group decision environment. Three MAS products were identified and ultimately ranked using the AHP.

Sarkis and Talluri [9] presented a decision framework that could aid members of the supply chain and a supply chain director in deciding which electronic commerce technology media and software is most suitable for the whole supply chain. The techniques used in this approach included both qualitative and quantitative measurements for the evaluation or justification of these systems. The framework used an integrative set of models based on the analytical hierarchy process and goal programming.

Wei et al. [10] presented a comprehensive framework for selecting a suitable enterprise resource planning (ERP) system using AHP based approach. A real world example was presented to demonstrate the feasibility of the framework. Mulebeke and Zheng [11] carried out a case study to introduce analytic network process (ANP) as a multiple attribute strategic decision making approach to help in the selection of appropriate software to suit the product development process of a particular product.

Shyur [12] proposed different evaluation criteria and the related attributes. The criteria (and the attributes) are: cost (license fee, modular pricing, maintenance, documentation, consultant fee, resource utilization, conversion cost, etc.), supplier's support (vendor responsiveness, consulting, hot line, training, technical support personnel, continuing enhancement, time sharing access, warranty, documentation, financial stability, local branch office, third vendor support, growth of customer base, active $\mathrm{R} \& \mathrm{D}$, etc.), technological risk (non-robust and incomplete packages, complex and undefined, COTSto-legacy-system interfaces, middleware technology bugs, poor custom code, and poor system performance, software maturity, hardware maturity, etc.), closeness of fit to the company's business (main target, included functionality, etc.), ease of implementation (shorter implementation time, user friendliness, multi- site implementation, etc.), flexibility to easy change as the company's business changes (adaptability, openness for customer development, openness for working with other systems, etc.) and system integration (internal connectivity, external connectivity, etc.). Shyur (2006) modeled COTS evaluation problem and proposed a five-phase COTS selection model combining the techniques of analytic network process (ANP) and modified TOPSIS. ANP was used to determine the relative weights of multiple attributes. The modified TOPSIS approach was used to rank the competing COTS products in terms of their overall performance. However, the TOPSIS method can't deal with qualitative criteria.

Otamendi et al. [13] suggested a suitable software that will help not only with the scheduling of resources but also with their real time control during normal operations of an airport in Spain. The selection process of the software was based on the study of the capabilities of the commercial and general-purpose simulation and visualization tools available as well as on the quantification of user requirements and the development of trial versions. AHP method was used to choose the platform, which was composed of a simulation model developed in JAVA and two visualization screens, one in JAVA and the other in Visual Basic.

Even though certain methods, as described above, were proposed in the past to address the issue of selection of an appropriate software for a given manufacturing industrial application, these methods do not make a provision to consider the qualitative software selection criteria (i.e. quantitative values are not available). There is a need for a logical scientific method to guide user organizations in taking a proper decision. This paper aims to propose such a decision making method based on Preference Ranking Organization Method for Enrichment Evaluations (PROMETHEE) in conjunction with AHP for the problem of selecting appropriate software from among the alternatives.

\section{PROMETHEE Method}

PROMETHEE method was introduced by Brans et al. [14] and belongs to the category of outranking methods. Like all outranking methods, PROMETHEE proceeds to a pairwise comparison of alternatives in each single criterion in order to determine partial binary relations denoting the strength of preference of an alternative $a$ over alternative $b$. In the evaluation table, the alternatives are 
evaluated on different criteria. These evaluations involve mainly quantitative data. The implementation of PROMETHEE requires additional types of information, namely:

- information on the relative importance that is the weights of the criteria considered, and

- information on the decision maker preference function, which he/she uses when comparing the contribution of the alternatives in terms of each separate criterion.

It may be added here that the original PROMETHEE method can effectively deal mainly with quantitative criteria. However, there exists some difficulty in the case of qualitative criteria. In the case of a qualitative criterion (i.e. quantitative value is not available); a ranked value judgment on a fuzzy conversion scale is adopted in this paper. By using fuzzy set theory, the value of the criteria can be first decided as linguistic terms, converted into corresponding fuzzy numbers and then converted to the crisp scores. Rao (2007) had presented a logical approach based on the work of Cheng and Hwang (1992). The presented numerical approximation system systematically converts linguistic terms to their corresponding fuzzy numbers. It contains eight conversion scales and in the present work, an eleven-point scale is considered for better understanding and representation. Table 1 is suggested which represents the selection criterion on a qualitative scale using fuzzy logic, corresponding to the fuzzy conversion scale as shown in Figure 1 and helps the users in assigning the values. For more details, one can refer to Chen and Hwang (1992). Once a qualitative criterion is represented on a scale then the alternatives can be compared with each other on this criterion in the same manner as that for quantitative criteria.

The methodology presented in this paper for software selection in the manufacturing environment using improved PROMETHEE method is described below:

Step-1: Identify the selection criteria for the considered decision making problem of software selection and shortlist the alternative softwares on the basis of the identified

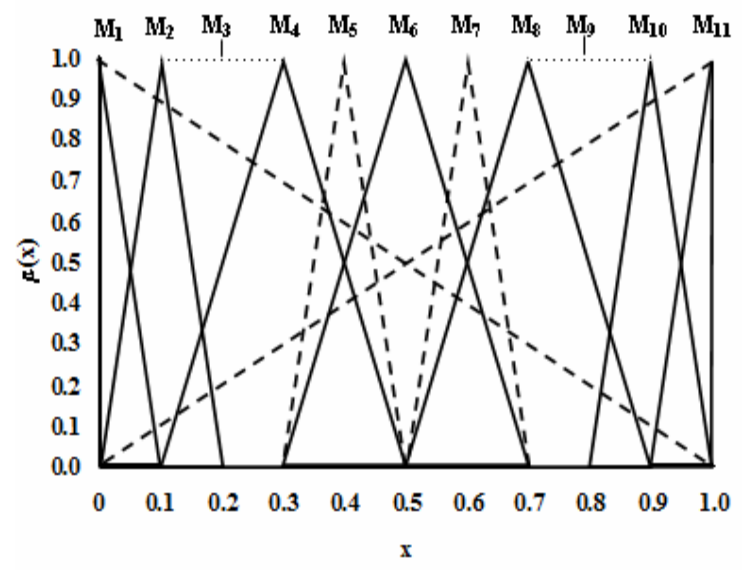

Figure 1. Linguistic terms to fuzzy numbers conversion criteria satisfying the requirements. A quantitative or qualitative value or its range may be assigned to each identified criterion as a limiting value or threshold value for its acceptance for the considered application. An alternative software with each of its criterion, meeting the criterion, may be short-listed. The short-listed alternatives may then be evaluated using the proposed methodology.

The values associated with the criteria for different alternatives may be based on the available data or may be the estimations made by the decision maker or a group of decision makers. In the case of group decision making, the values estimated by the decision makers for different criteria for different alternatives may be different. In such cases, the value of a criterion for an alternative may be determined by averaging the estimated values given by the group of decision makers for that criterion for that alternative. The same averaging procedure may be carried out for other criteria. Alternately, the group may decide the values of criteria for different alternatives based on group consensus.

Step-2:

1) After short-listing the alternatives, prepare a decision table including the measures or values of all criteria for the short-listed alternatives.

2) The original PROMETHEE method has no systematic way of assigning the weights of relative importance to the criteria. Hence, use of analytic hierarchy process (AHP) is suggested in this paper to be used in conjunction with PROMETHEE for this purpose.

The steps are explained below:

a) Find out the relative importance of different criteria with respect to the objective. To do so, one has to construct a pair-wise comparison matrix using a scale of relative importance. The judgments are entered using the fundamental scale of the AHP. An attribute compared with it is always assigned the value 1 so the main diagonal entries of the pair-wise comparison matrix are all 1 . The numbers 3, 5, 7, and 9 correspond to the verbal judgments 'moderate importance', 'strong importance', 'very strong importance', and 'absolute importance' (with 2, 4, 6, and 8 for compromise between the previous values). Assuming $M$ criteria, the pair-wise comparison of attribute $\mathrm{i}$ with attribute $\mathrm{j}$ yields a square matrix $A 1$ where $r_{i j}$ denotes the comparative importance of attribute $i$ with respect to attribute $j$. In the matrix, $r_{i j}=1$ when $i=$ $j$ and $r_{j i}=1 / r_{i j}$

$$
\begin{gathered}
\text { Criterion } \\
1 \\
2 \\
3 \\
\cdots \\
\cdots \\
M
\end{gathered}\left[\begin{array}{cccccc}
1 & 2 & 3 & \cdots & \cdots & M \\
r_{11} & r_{12} & r_{13} & \cdots & \cdots & r_{1 M} \\
r_{21} & r_{22} & r_{23} & \cdots & \cdots & r_{2 M} \\
r_{31} & r_{32} & r_{33} & \cdots & \cdots & r_{3 M} \\
\cdots & \cdots & \cdots & \cdots & \cdots & \cdots \\
\cdots & \cdots & \cdots & \cdots & \cdots & \cdots \\
r_{M 1} & r_{M 2} & r_{M 3} & \cdots & \cdots & r_{M M}
\end{array}\right]
$$


b) Find the relative normalized weight $\left(W_{j}\right)$ of each attribute by i) calculating the geometric mean of $i^{\text {th }}$ row and ii) normalizing the geometric means of rows in the comparison matrix. This can be represented as

$$
G M_{i}=\left\{\prod_{j=I}^{M} r_{i j}\right\}^{I / M}
$$

and

$$
W_{j}=G M_{i} / \sum_{i=1}^{M} G M_{i}
$$

The geometric mean method of AHP is used in the present work to find out the relative normalized weights of the attributes because of its simplicity and easiness to find out the maximum Eigen value and to reduce the inconsistency in judgments.

c) Calculate matrix $A 3$ and $A 4$ such that $A 3=A 1 \times A 2$ and $A 4=A 3 / A 2$, where $A 2=\left[W_{1}, W_{2}, \ldots \ldots, W_{M}\right]^{T}$. Each element of $A 4$ is obtained by dividing each element of $A 3$ by the corresponding element of $A 2$.

d) Find out the maximum eigen value $\lambda_{\max }$ (i.e. the average of matrix $A 4$ ).

e) Calculate the consistency index $\mathrm{CI}=\left(\lambda_{\max }-M\right) /(M$ $-1)$. The smaller the value of CI, the smaller is the deviation from the consistency.

f) Obtain the random index (RI) for the number of attributes used in decision making [15].

g) Calculate the consistency ratio $\mathrm{CR}=\mathrm{CI} / \mathrm{RI}$. Usually, a CR of 0.1 or less is considered as acceptable and it reflects an informed judgment that could be attributed to the knowledge of the analyst about the problem under study.

Step-3: After calculating the weights of the criteria using AHP method, the next step is to have the information on the decision maker preference function, which he/she uses when comparing the contribution of the alternatives in terms of each separate criterion.

The preference function $\left(P_{i}\right)$ translates the difference between the evaluations obtained by two alternatives ( $a 1$ and $a 2$ ) in terms of a particular criterion, into a preference degree ranging from 0 to 1 . Let $P_{i, \text { ala } 2}$ be the preference function associated to the criterion $c_{i}$.

$$
\begin{aligned}
P_{i, a l a 2} & =G_{i}\left[c_{i}(a 1)-c_{i}(a 2)\right] \\
0 & \leq P_{i, a l a 2} \leq 1
\end{aligned}
$$

where $G_{i}$ is a non-decreasing function of the observed deviation (d) between two alternatives $a 1$ and $a 2$ over the criterion $c_{i}$. In order to facilitate the selection of a specific preference function, six basic types were proposed $[14,17]$. These include "usual function", "linear function", "U-shape function", "V-shape function", "level function" and "Gaussian function". Preference "usual function" which is equal to the simple difference between the values of the criterion $c_{i}$ for alternatives $a l$ and $a 2$ is adapted in this paper because of its simplicity. For other preference functions, no more than two parameters (threshold $q, p$ or $s$ ) have to be fixed. Indifference threshold ' $q$ ' is the largest deviation to consider as negligible on that criterion and it is a small value with respect to the scale of measurement. Preference threshold ' $p$ ' is the smallest deviation to consider decisive in the preference of one alternative over another and it is a large value with respect to the scale of measurement. Gaussian threshold ' $s$ ' is only used with the Gaussian preference function. It is usually fixed as an intermediate value between indifference and a preference threshold.

Let us suppose that the decision maker has specified a preference function $P_{i}$ and weight $w_{i}$ for each criterion $c_{i}$ $(i=1,2, \ldots, M)$ of the problem. The multiple criteria preference index $\Pi_{\text {ala } 2}$ is then defined as the weighted average of the preference functions $P_{i}$ :

$$
\Pi_{\text {ala2 }}=\sum_{i=1}^{M} w_{i} P_{i, \text { ala } 2}
$$

$\Pi_{a 1 a 2}$ represents the intensity of preference of the decision maker of alternative al over alternative $a 2$, when considering simultaneously all the criteria. Its value ranges from 0 to 1 . This preference index determines a valued outranking relation on the set of actions. As an example, the schematic calculation of the preference indices for a problem consisting of 3 alternatives and 4 criteria is given in Figure 2.

For PROMETHEE outranking relations, the leaving flow, entering flow and the net flow for an alternative ' $a$ ' belonging to a set of alternatives $A$ are defined by the following equations:

$$
\begin{gathered}
\varphi^{+}(a)=\sum_{x \varepsilon A} \Pi_{x a} \\
\varphi^{-}(a)=\sum_{x \varepsilon A} \Pi_{a x} \\
\varphi(a)=\varphi^{+}(a)-\varphi^{-}(a)
\end{gathered}
$$

$\varphi^{+}(a)$ is called the leaving flow, $\varphi^{-}\left(a_{i}\right)$ is called the entering flow and $\varphi\left(a_{i}\right)$ is called the net flow. $\varphi^{+}(a)$ is the

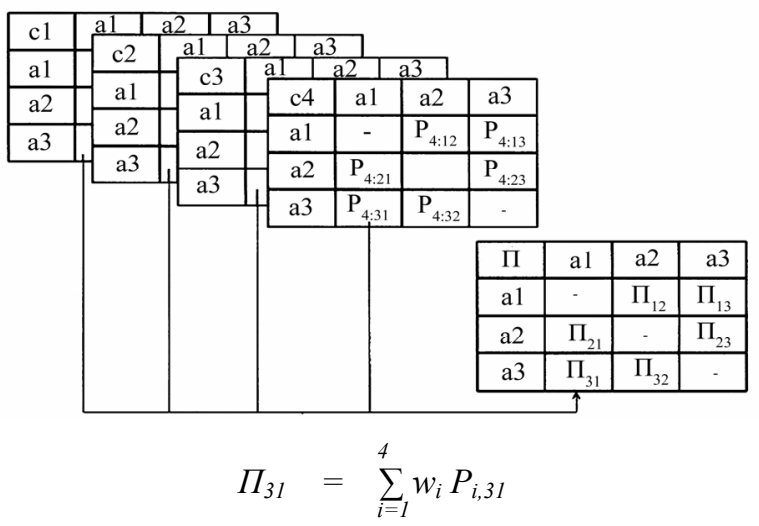

Figure 2. Preference indices for a problem consisting of 3 alternatives and 4 criteria 
measure of the outranking character of $a$ (i.e. dominance of alternative $a$ over all other alternatives) and $\varphi^{-}(a)$ gives the outranked character of $a$ (i.e. degree to which alternative $a$ is dominated by all other alternatives). The net flow, $\varphi(a)$, represents a value function, whereby a higher value reflects a higher attractiveness of alternative $a$. The net flow values are used to indicate the outranking relationship between the alternatives. For example, for each alternative $a$, belonging to the set $A$ of alternatives, $\Pi_{a l a 2}$ is an overall preference index of $a 1$ over $a 2$, taking into account all the criteria, $\varphi^{+}(a)$, and $\varphi^{-}(a)$. Alternative $a 1$ outranks $a 2$ if $\varphi(a 1)>\varphi(a 2)$ and $a 1$ is said to be indifferent to $a 2$ if $\varphi(a 1)=\varphi(a 2)$. The proposed decision making framework using PROMETHEE method provides a complete ranking of the alternatives from the best to the worst one using the net flows. A computer program is developed in the present work in MATLAB environment that can be used for improved PROMETHEE calculations. Any number of alternatives and the criteria can be considered and the time required for computation is less.

Now an example of software selection is considered to demonstrate the applicability of the proposed PROMETHEE method.

\section{Example}

Shyur [12] modeled COTS evaluation problem and proposed a five-phase COTS selection model combining the techniques of analytic network process (ANP) and modified TOPSIS. The modified TOPSIS approach was used to rank the competing COTS products in terms of their overall performance. To illustrate the approach for the COTS evaluation problem, an empirical study of a real case of 'off-line production data analysis system' selection problem for implementation in an electronic company was conducted. To conduct the empirical study, enough information was gathered through interviews with users and managers, observation of current operation process, and analysis of the systems documentation to develop some general ideas for the to-be system. Next, the screening criteria were created. Thus, four alternative softwares and seven criteria were considered. The criteria considered were: cost (CO), supplier's support (SS), ease of implementation (EI), closeness of fit to the company's business (FB), flexibility to easy change as the company's business changes (FC), technological risk (TR), and system integration (SI). Here each criterion is a broader one and includes many factors. All seven criteria were considered as beneficial (i.e. higher values are desirable) The values of criterion TR were so decided by Shyur [12] that TR was considered as a beneficial criterion. Shyur [12] had established the decision matrix by comparing alternative software under each of the criteria separately. A set of crisp values within the range from 1 to 10 to represent the performance of each alternative
Table 1. Values of software selection criterion

\begin{tabular}{cc}
\hline Qualitative measures of selection & Assigned value \\
criterion & \\
\hline Exceptionally low & 0.045 \\
Extremely low & 0.135 \\
Very low & 0.255 \\
Low & 0.335 \\
Below average & 0.410 \\
Average & 0.500 \\
Above average & 0.590 \\
High & 0.665 \\
Very high & 0.745 \\
Extremely high & 0.865 \\
Exceptionally high & 0.955 \\
\hline
\end{tabular}

Table 2. Normalized values of software selection criteria [12]

\begin{tabular}{cccccccc}
\hline Software & CO & SS & EI & FB & FC & TR & SI \\
\hline $\mathrm{A}_{1}$ & 0.55 & 0.70 & 0.39 & 0.64 & 0.61 & 0.30 & 0.55 \\
$\mathrm{~A}_{2}$ & 0.46 & 0.35 & 0.55 & 0.40 & 0.41 & 0.69 & 0.39 \\
$\mathrm{~A}_{3}$ & 0.28 & 0.35 & 0.63 & 0.32 & 0.30 & 0.59 & 0.39 \\
$\mathrm{~A}_{4}$ & 0.64 & 0.52 & 0.39 & 0.56 & 0.61 & 0.30 & 0.63 \\
\hline
\end{tabular}

software with respect to each criterion were assigned. After the decision matrix was determined, the matrix was normalized and the normalized values were as given in Table 2.

Now, various steps of the proposed PROMETHEE method for software selection are explained below.

Step-1: The seven criteria used to evaluate the four short-listed alternative softwares included cost (CO), supplier's support (SS), ease of implementation (EI), closeness of fit to the company's business (FB), flexibility to easy change as the company's business changes (FC), technological risk (TR), and system integration (SI). Table 2 presents the normalized data of the software selection criteria.

Step-2:

1) A decision table including the measures or values of all criteria for the short-listed alternatives is prepared and it corresponds to Table 2 .

2) The weights of the seven criteria are obtained by using the analytic hierarchy process (AHP) method using the following relations of relative importance. It may be added here that the assigned relative importance values are for demonstration purpose only and, in actual practice, these values are to be judiciously decided by the user organizations.

\begin{tabular}{llllllll|}
\multicolumn{1}{l}{} & CO & SS & EI & FB & FC & TR & SI \\
CO & 1 & $1 / 2$ & 5 & 3 & 7 & 2 & 4 \\
$\mathrm{SS}$ & 2 & 1 & 7 & 4 & 8 & 3 & 5 \\
$\mathrm{EI}$ & $1 / 5$ & $1 / 7$ & 1 & $1 / 3$ & 2 & $1 / 5$ & $1 / 2$ \\
$\mathrm{FB}$ & $1 / 3$ & $1 / 4$ & 3 & 1 & 4 & $1 / 2$ & 2 \\
$\mathrm{FC}$ & $1 / 7$ & $1 / 8$ & $1 / 2$ & $1 / 4$ & 1 & $1 / 4$ & $1 / 3$ \\
$\mathrm{TR}$ & $1 / 2$ & $1 / 3$ & 5 & 2 & 4 & 1 & 3 \\
$\mathrm{SI}$ & $1 / 4$ & $1 / 5$ & 2 & $1 / 2$ & 3 & $1 / 3$ & 1
\end{tabular}

The weights are obtained by following the steps of AHP method for the criteria CO, SS, EI, FB, FC, T and 
SI are $0.242,0.360,0.042,0.102,0.030,0.157$ and 0.067 respectively and the consistency ratio (CR) is less than 0.1 . Thus, there is good consistency Shyur et al. [12] obtained the same weights using ANP method in their approach. To compare the results of the proposed PROMETHEE method, the same weights have been adopted in the present example.

Step-3: After calculating the weights of the criteria using AHP method, the next step is to have the information on the decision maker preference function, which he/she uses when comparing the contribution of the alternatives in terms of each separate criterion. Let the decision maker uses the preference "usual function". The preference function $\left(P_{i}\right)$ translates the difference between the evaluations obtained by two alternatives ( $a 1$ and $a 2)$ in terms of a particular criterion, into a preference degree ranging from 0 to 1 . If two alternatives have a difference $\mathrm{d} \neq 0$ in criterion $c_{j}$, then a preference value $P_{i}$ ranging between 0 and 1 will be assigned to the 'better' alternative whereas the 'worse' alternative receives a value 0 . If two criteria have a zero difference, they are indifferent which results in an assignment of 0 to both alternatives. The pairwise comparison of criterion CO gives the matrix shown in Table 3. The alternative software having comparatively high value of $\mathrm{CO}$ is said to be 'better' than the other. Similarly, the pairwise comparisons of the four alternatives with respect to other criteria are made, but not shown here for space reasons.

Table 4 is prepared which shows the resulting preference indices as well as leaving, entering, and net flows of the alternatives of Table 2. The preference indices are calculated based on Equation (9) and the leaving, entering, and net flows of the alternatives are calculated based on Equations (7), (8), and (9) respectively. From the values of software ranking, software $A_{1}$ is understood as the best choice among the considered software alternatives

Table 3. Preference values resulting from the pairwise comparisons of the alternatives $A_{1}$ to $A_{4}$ with respect to criterion $\mathrm{CO}$

\begin{tabular}{ccccc}
\hline Software & $\mathrm{A}_{1}$ & $\mathrm{~A}_{2}$ & $\mathrm{~A}_{3}$ & $\mathrm{~A}_{4}$ \\
\hline $\mathrm{A}_{1}$ & --- & 1 & 1 & 0 \\
$\mathrm{~A}_{2}$ & 0 & --- & 1 & 0 \\
$\mathrm{~A}_{3}$ & 0 & 0 & --- & 0 \\
$\mathrm{~A}_{4}$ & 1 & 1 & 1 & --- \\
\hline
\end{tabular}

Table 4. Resulting preference indices as well as leaving, entering, and net flows

\begin{tabular}{cccccccc}
\hline$\Pi$ & $\mathrm{A}_{1}$ & $\mathrm{~A}_{2}$ & $\mathrm{~A}_{3}$ & $\mathrm{~A}_{4}$ & $\varphi^{+}(a)$ & $\varphi(a)$ & Ranking \\
\hline $\mathrm{A}_{1}$ & --- & 0.799 & 0.799 & 0.462 & 2.06 & 1.359 & 1 \\
$\mathrm{~A}_{2}$ & 0.197 & --- & 0.529 & 0.197 & 0.923 & -0.715 & 3 \\
$\mathrm{~A}_{3}$ & 0.197 & 0.04 & --- & 0.197 & 0.434 & -1.693 & 4 \\
$\mathrm{~A}_{4}$ & 0.307 & 0.799 & 0.799 & --- & 1.905 & 1.049 & 2 \\
$\varphi(a)$ & 0.701 & 1.638 & 2.127 & 0.856 & & & \\
\hline
\end{tabular}

for the given software selection problem under consideration. However, these results differ from the results presented by Shyur [12]. Using modified TOPSIS procedure, Shyur [12] obtained the following ranking for the considered softwares: $\mathrm{A}_{4}=0.652, \mathrm{~A}_{1}=0.645, \mathrm{~A}_{2}=0.433$, and $\mathrm{A}_{3}=0.236$. However, Shyur [12] had committed some mistakes in computing the closeness coefficient values of the alternative software. Removal of those mistakes would lead to the following ranking: $A_{1}=0.6908, A_{4}=$ $0.5520, \mathrm{~A}_{2}=0.3556$ and $\mathrm{A}_{3}=0.2261$. This also suggests $\mathrm{A}_{1}$ as the best choice. Thus, the results proposed by PROMETHEE method are justified and reliable. It may be added here that Shyur [12] had not considered any qualitative criteria in the considered problem. The TOPSIS method used by Shyur [12] can't deal with qualitative criteria. However, the proposed PROMETHEE method can easily deal with such qualitative criteria using Table 1 .

\section{Conclusions}

The selection of suitable software would increase the competitive advantage of an industry. This paper has presented the details of a decision making framework for software selection in manufacturing industries using PROMETHEE method in conjunction with AHP method. The proposed PROMETHEE method is a fairly simple and therefore transparent for decision makers and stakeholders who are often non-experts. Furthermore, the paper suggests ranked value judgments on a fuzzy conversion scale to represent the qualitative software selection criterion. The proposed decision making framework using PROMETHEE method can be extended to any type of decision making problem involving any number of criteria and alternatives.

\section{Acknowledgement}

The authors acknowledge the financial support of Council of Scientific and Industrial Research (CSIR) New Delhi, India to carry out this work.

\section{REFERENCES}

[1] L. Mikhailov and M. G. Singh, "Fuzzy analytic network process and its application to the development of decision support systems," IEEE Transactions on Systems, Man, and Cybernetics, Part C. Applications and Reviews, Vol. 33, No. 1, pp. 33-41, 2003.

[2] R. Santhanam and G. J. Kyparisis, "A decision model for interdependent information system project selection," European Journal of Operational Research, Vol. 89, No. 2, pp. 380-399, 1996.

[3] R. Santhanam and G. J. Kyparisis, "A multiple criteria decision model for information system project selection," Computers \& Operations Research, Vol. 22, No. 8, pp. 807-818, 1995. 
[4] D. J. Carney and K. C. Wallnau, "A basis for evaluation of commercial software. information and software technology," Information and Software Technology, Vol. 40, No. 14, pp. 851-860, 1998.

[5] J. Sarkis and R. P. Sundarraj, "Factors for strategic evaluation of enterprise information technologies," International Journal of Physical Distribution \& Logistics Management, Vol. 30, No. 3, pp. 196-220, 2000.

[6] M. A. Badri, D. Davis, and D. Davis, "A comprehensive 0-1 goal programming model for project selection," International Journal of Project Management, Vol. 19, No. 4, pp. 243-252, 2001.

[7] M. Morisio, C. B. Seaman, V. R. Basili, A. T. Parra, S. E. Kraft, and S. E. Condon, "COTS-based software development: processes and open issues," Journal of Systems and Software, Vol. 61, No. 3, pp. 189-199, 2002.

[8] V. S. Lai, K. W. Bo, and W. Cheung, "Group decision making in a multiple criteria environment: A case using the AHP in software selection," European Journal of Operational Research, Vol. 137, No. 1, pp. 34-144, 2002.

[9] J. Sarkis and S. Talluri, "Evaluating and selecting e-commerce software and communication systems for a supply chain," European Journal of Operational Research, Vol. 159, No. 2, pp. 318-329, 2004.

[10] C. C. Wei, C. F. Chien, and M. J. J. Wang, "An AHPbased approach to ERP system selection," International Journal of Production Economics, Vol. 96, No. 1, pp. 47-
$62,2005$.

[11] J. A. W. Mulebeke, L. Zheng, "Analytical network process for software selection in product development," Journal of Engineering and Technology Management, Vol. 23, No. 4, pp. 337-352, 2006.

[12] H. J. Shyur, "COTS evaluation using modified TOPSIS and ANP," Applied Mathematics and Computation, Vol. 177, No. 1, pp. 251-259, 2006.

[13] J. Otamendi, J. M. Pastor, and A. Garc1'a, "Selection of the simulation software for the management of the operations at an international airport," Simulation Modelling Practice and Theory, Vol. 16, No. 8, pp. 1103-1112, 2008.

[14] J. P. Brans, B. Mareschal, and P. Vincke, "PROMETHEE: A new family of outranking methods in multicriteria analysis," Operational Research, Vol. 3, pp. 477-490. 1984.

[15] T. L. Saaty, "Fundamentals of decision making and priority theory with AHP," RWS Publications, Pittsburg, 2000.

[16] R. V. Rao, "Decision making in the manufacturing environment using graph theory and fuzzy multiple attribute decision making methods," Springer-Verlag, London, 2007.

[17] O. Marinoni, "A stochastic spatial decision support system based on PROMETHEE," International Journal of Geographical Information Science, Vol. 19, No. 1, pp. 51-68, 2005. 